\title{
Charge transfer mechanism for the formation of metallic states at the $\mathrm{KTaO}_{3} / \mathrm{SrTiO}_{3}$ interface
}

\author{
S. Nazir, N. Singh, and U. Schwingenschlögl* \\ KAUST, Physical Science \& Engineering Division, Thuwal 23955-6900, Kingdom of Saudi Arabia
}

(Received 9 February 2011; published 29 March 2011)

\begin{abstract}
The electronic and optical properties of the $\mathrm{KTaO}_{3} / \mathrm{SrTiO}_{3}$ heterointerface are analyzed by the full-potential linearized augmented plane-wave approach of density functional theory. Optimization of the atomic positions points at subordinate changes in the crystal structure and chemical bonding near the interface, which is due to a minimal lattice mismatch. The creation of metallic interface states thus is not affected by structural relaxation but can be explained by charge transfer between transition metal and oxygen atoms. It is to be expected that a charge transfer is likewise important for related interfaces such as $\mathrm{LaAlO}_{3} / \mathrm{SrTiO}_{3}$. The $\mathrm{KTaO}_{3} / \mathrm{SrTiO}_{3}$ system is ideal for disentangling the complex behavior of metallic interface states, since almost no structural relaxation takes place.
\end{abstract}

DOI: 10.1103/PhysRevB.83.113107

PACS number(s): 73.20.-r, 71.15.Mb

During the past decade, heterointerfaces based on perovskite transition metal oxides have attracted a great deal of attention due to the discovery of remarkable electronic and magnetic properties. They are of special importance for device applications, such as field effect transistors, bipolar transistors, and light emitting diodes. ${ }^{1,2}$ Ohtomo and $\mathrm{Hwang}^{3}$ have reported on metallicity at the interface between $\mathrm{LaAlO}_{3}$ and $\mathrm{SrTiO}_{3}$, two insulators with sizable band gaps of 5.6 and 3.2 $\mathrm{eV}$, respectively. The creation of metallic states is intimately connected to the structural relaxation. ${ }^{4}$ The high mobility of the charge carriers at this interface has initiated many investigations, ${ }^{5,6}$ as did the discovery of superconductivity at low temperature. ${ }^{7}$ However, despite huge experimental and theoretical efforts, many aspects of the physical picture of the $\mathrm{LaAlO}_{3} / \mathrm{SrTiO}_{3}$ (LAO/STO) interface remain open. ${ }^{8-13}$ To a large extent, this appears to be the result of a complex interplay between structural and electronic reconstructions as well as defects. ${ }^{14}$

An essential ingredient of the electronic reconstruction is the transfer of charge between different orbitals, i.e., alterations of the orbital occupations, which can modify the electronic properties of transition metal oxides significantly. ${ }^{15}$ An interesting system to disentangle the effects of the charge transfer from other parameters is the $\mathrm{KTaO}_{3} / \mathrm{SrTiO}_{3}$ (KTO/STO) heterointerface. KTO has an insulting band gap of $3.75 \mathrm{eV}$ and does not show the ferroelectric phase transition that is common for perovskites. ${ }^{16,17}$ The band gap of STO amounts to $3.2 \mathrm{eV}$. Its electronic, transport, ferroelectric, catalytic, and dielectric properties have been widely studied. ${ }^{18-21}$ Interestingly, the lattice match of KTO with STO is much better than that of LAO with STO. As a consequence, KTO is a promising material for application as an insulator in STO-based field-effect devices. Recently, Kalabukhov et al. ${ }^{22}$ have investigated the KTO/STO interface experimentally and found close relations to the LAO/STO interface, even though at the $(\mathrm{KO})^{-}\left(\mathrm{TiO}_{2}\right)^{0}$ contact 0.5 holes per unit cell are transferred, while at the $(\mathrm{LaO})^{+}(\mathrm{TiO} 2)^{0}$ contact 0.5 electrons are transferred.

The electronic structures of bulk KTO and STO are well studied experimentally and theoretically, which does not apply to the STO/KTO interface. In this context, it is the aim of our present study to clarify the interaction between the component materials in the STO/KTO heterointerface. We deduce a general mechanism based on charge transfer between the transition metal and oxygen atoms, which is expected to be of broad importance for related interfaces such as $\mathrm{LaAlO}_{3} / \mathrm{SrTiO}_{3}$.

The calculations are performed by the full-potential linearized augmented plane-wave (FP-LAPW) method in the framework of density functional theory, ${ }^{23}$ using the WIEN $2 \mathrm{~K}$ code. $^{24}$ The exchange-correlation potential is computed in the local density approximation. Fully relativistic effects are taken into account for the core states, while the scalar relativistic approximation is used for the valence states. Therefore, the effects of spin-orbit coupling are neglected. We have fully optimized the crystal structure of the KTO/STO interface by minimizing the atomic forces. For the wave-function expansion inside the atomic spheres, a maximum value of $l_{\max }=12$ is chosen and a plane-wave cutoff of $R_{\mathrm{mt}} K_{\max }=7.0$ with $G_{\max }=18$ employed. In all our calculations, we ensure a total energy convergence of $3 \times 10^{-5} \mathrm{eV}$.

For the self-consistent calculations, a $19 \times 19 \times 1 k$-space grid containing $55 k$ points within the irreducible wedge of the Brillouin zone (IBZ) is used. Our basis set comprises the valence states $\mathrm{K} 4 s, 4 p, 3 d$; $\mathrm{Sr} 5 s, 5 p$; Ta $6 s, 5 d$; Ti $4 s, 4 p$, $3 d$; and $\mathrm{O} 3 s, 2 p$. The muffin-tin sphere radii $R_{\mathrm{mt}}$ (in atomic units) are set to values of 2.5 for both $\mathrm{K}$ and $\mathrm{Sr}, 1.98$ for both $\mathrm{Ta}$ and $\mathrm{Ti}$, and 1.75 for $\mathrm{O}$. To provide a reliable Brillouin zone integration for the optical properties, a $k$-space grid of $132 k$ points in the IBZ is used. The convergence with respect to the fineness of the $k$-space grid is reached by refining the mesh until no appreciable change in the energy or optical properties is left. A Gaussian broadening of $0.1 \mathrm{eV}$ is applied to simulate the experimental finite lifetime effects.

Both KTO and STO crystallize in cubic structures with space group $221(P m \overline{3} m) \cdot{ }^{25,26}$ The experimental lattice constants for cubic KTO and STO are 3.988 and $3.905 \AA$, respectively. ${ }^{27,28}$ The interface of the two perovskite compounds is modeled by a supercell of five layers of KTO attached to five layers of STO perpendicular to the interface. By the employed periodic boundary conditions, the supercell contains two interfaces; we will focus on the $(\mathrm{KO})^{-}\left(\mathrm{TiO}_{2}\right)^{0}$ interface. The structural setup is illustrated in Fig. 1. For the supercell, we use an average lattice constant of $3.946 \AA$ for both the KTO and STO regions. We have increased the thickness of 


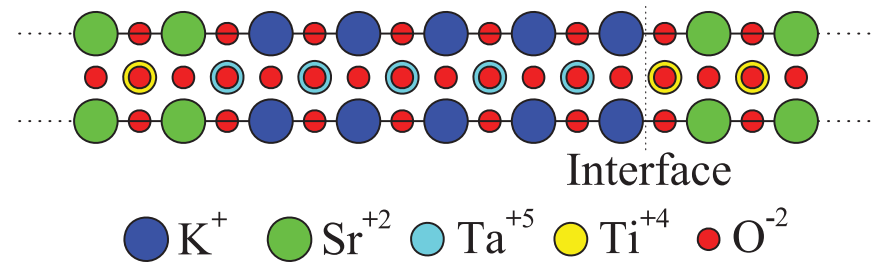

FIG. 1. (Color online) Schematic representation of the KTO/STO interface with the KTO on the left side and the STO on the right side. Large and medium spheres represent $\mathrm{K} / \mathrm{Sr}$ and $\mathrm{Ta} / \mathrm{Ti}$ atoms, respectively. Small spheres denote $\mathrm{O}$ atoms.

the KTO and STO slabs until the bulk behavior was reproduced in the central layers.

Turning to the results of our calculations, it is observed that in the vicinity of the interface, the electronic structure depends critically on structural details. For this reason, it is necessary to optimize the atomic structure by atomic force minimization. However, due to the minimal lattice mismatch between the component materials, the structural optimization results only in very small modifications of the bond lengths near the interface. The K-K and $\mathrm{Sr}-\mathrm{Sr}$ (3.990 $\AA$ in the bulk) bond lengths remain virtually the same and also the changes of the $\mathrm{K}-\mathrm{Ta}$ and $\mathrm{Sr}-\mathrm{Ti}$ ( $3.455 \AA$ in the bulk) bond lengths are small: $3.453 \AA$ for $\mathrm{K}-\mathrm{Ta}$ and $3.455 \AA$ for $\mathrm{Sr}-\mathrm{Ti}$ at the interface. The same applies to the K-O and $\mathrm{Sr}-\mathrm{O}(2.821 \AA$ in the bulk) bond lengths, which become $2.823 \AA$. The Ta-O and Ti-O $(2.00 \AA$ in the bulk) bond lengths slightly shrink at the interface to 1.989 $\AA$ for Ta-O and $1.992 \AA$ for Ti-O.

Figure 2 shows the calculated partial densities of states (DOS), which are obtained by projections on the $\mathrm{Ta} / \mathrm{Ti}$ and $\mathrm{O}$ layers at the interface. They are compared to corresponding results for bulk KTO and bulk STO to establish a qualitative description of the atomic and orbital origin of the different electronic states. Panels (a) and (b) of Fig. 2 clearly indicate a semiconducting behavior of both the Ta $5 d$ and Ti $3 d$ states with an energy gap of approximately $1.7 \mathrm{eV}$, which is clearly less than expected from experiment. We note that such an underestimation of semiconducting band gaps is a well-known drawback of the local density approximation. Turning to the interface, we observe that the electronic states shift considerably, by about $2 \mathrm{eV}$, to lower energy. This shift affects both the valance and conduction bands by a similar amount. Due to the energetic downshift, the conduction states cross the Fermi energy $\left(E_{F}\right)$ and consequently leave the interface metallic. The metallic nature perfectly resembles the experimental findings of Kalabukhov and co-workers. ${ }^{22} \mathrm{We}$ mention that these authors have expressed concerns about a possible $\mathrm{K}$ deficiency of the samples, which may invalidate their conclusions. However, according to our results even the perfect interface exhibits a metallic state.

Moreover, as is shown in panels (c) and (d) of Fig. 2, the $\mathrm{O} 2 p$ states shift to higher energy as compared to the situation in bulk KTO and STO. Therefore, also O $2 p$ states appear at $E_{F}$. The metallic interface states are thus mainly due to the $\mathrm{O} 2 p$ and Ti $3 d$ states of atoms near the interface. In addition, there is a small contribution of the Ta $5 d$ states. The number of unoccupied $\mathrm{O} 2 p$ states is larger for the $\mathrm{O}$ atoms in the $\mathrm{TiO}_{2}$ interface layer than for those in the $\mathrm{KO}$ interface layer, see Figs. 2(c) and 2(d). These findings are confirmed by the band structure, which we show on the right-hand side of Fig. 2.

We next address the origin of the interface metallicity. According to our previous discussion, alterations of the bond
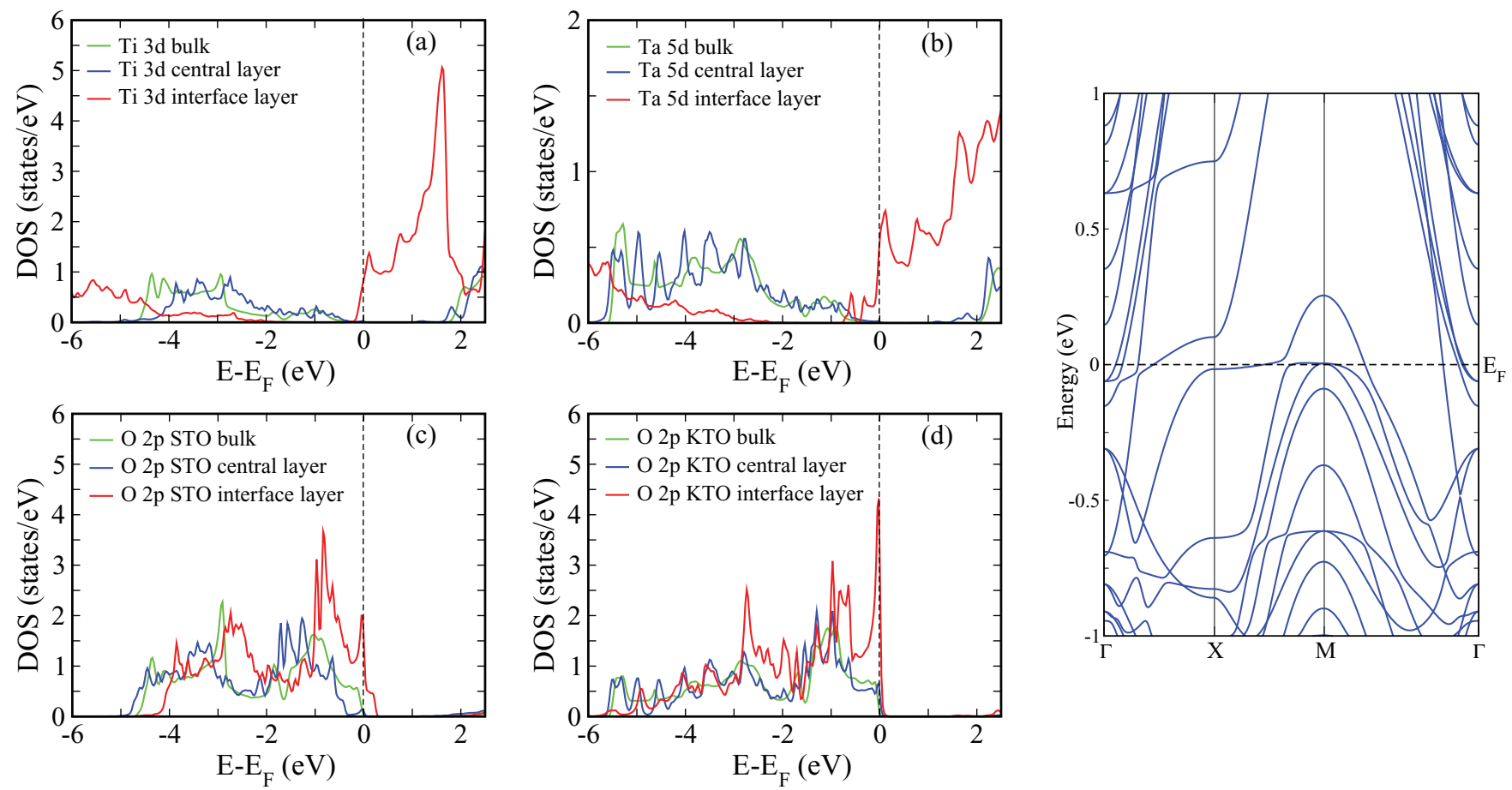

FIG. 2. (Color online) Projected Ti, Ta, and O densities of states obtained for the fully relaxed KTO/STO interface, as compared to the bulk KTO and STO compounds. In addition, the band structure of the interface system is shown. 
lengths and bond angles at the interface are subordinate. Thus, an alteration of the chemical bonding due to a modified crystal structure must be excluded as the origin of the metallic states. In this respect, the KTO/STO heterointerface behaves very differently as compared to related systems, such as the LAO/STO interface, due to the almost optimal lattice match between KTO and STO. As a consequence, the present system is an ideal candidate for studying the electronic interface relaxation without the complication of a simultaneous structure relaxation. However, the question about the origin of the metallic states still remains. A careful analysis of the calculated charges within the muffin-tin spheres paves the way to a clarification of the situation.

We observe that in the vicinity of the interface, both transition metals (TM), i.e., Ti and Ta, gain charge while the $\mathrm{O}$ atoms lose charge. We note that due to interstitial electronic states, outside the muffin-tin spheres, this charge transfer in reality is stronger than reflected by the following values. While the muffin-tin charges in the bulk compounds amount to 19.305 for $\mathrm{Ti}, 7.547$ for $\mathrm{O}$, and 68.857 for $\mathrm{Ta}$, we find the subsequent values for the atoms next to the interface: 19.322 for $\mathrm{Ti}$, 7.507 for $\mathrm{O}$ next to $\mathrm{Ti}, 7.639$ for $\mathrm{O}$ next to $\mathrm{Ta}$, and 68.905 for Ta. For $\mathrm{O}$ within the $(\mathrm{KO})^{-}$layer forming the interface, the corresponding charge is 7.560. Therefore, the occupations of the $\mathrm{Ti}$ and $\mathrm{Ta}$ orbitals grow by 0.017 and 0.048 electrons, respectively, and the conduction bands become partially filled. This explains the shift of the DOS to lower energy. In contrast, $\mathrm{O}$ atoms next to $\mathrm{Ti}$ and $\mathrm{Ta}$ lose 0.040 and 0.002 electrons, respectively. This clarifies the shift of the O $2 p$ DOS to higher energy, which leaves the $\mathrm{O}$ orbitals partially empty. Finally, for the $\mathrm{O}$ within the central $(\mathrm{KO})^{-}$layer, we expect an electron count given by the average of the KTO and STO bulk values, i.e., of 7.594. Hence, this site experiences a loss of 0.034 electrons and consequently also develops metallic states. In the DOS, compare Figs. 2(c) and 2(d), the metallic O $2 p$ contributions show up as sharp peaks right at $E_{F}$.

The described modifications of the orbital occupations can be related to significant changes in the TM-O bonding. In general, a TM-O bond is characterized by a very strong transfer of charge from the TM to the $\mathrm{O}$ atoms. Therefore, a reduced charge transfer, as observed for the KTO/STO heterointerface, indicates a weakening of the TM-O bonding. This could be explained by the broken symmetry inherent to the Ti-O-Ta bond across the interface. Concerning the spatial extension of the metallic interface states, we find that they are restricted to a narrow region around the interface. Charge transfer effects mainly occur within the first $\mathrm{TiO}_{2} / \mathrm{TaO}_{2}$ layer off the interface, which shows a clearly metallic nature, see Fig. 2. The second $\mathrm{TiO}_{2} / \mathrm{TaO}_{2}$ layer, in contrast, is at the edge of metallicity, with only a few metallic states left. Regions even further off the interface finally are found to be insulating. As a consequence, the electron gas formed at the KTO/STO heterointerface is restricted to an area of about $12 \AA$ thickness (perpendicular to the interface).

Figure 3 depicts the imaginary part of the dielectric function, $\operatorname{Im}(\epsilon)$, and the reflectivity of the STO/KTO interface. For comparison, we also present corresponding data for bulk STO and bulk KTO. Our results clearly reflect the metallic nature of the heterointerface (increased reflectivity in the low-energy range), while both bulk STO and KTO show an
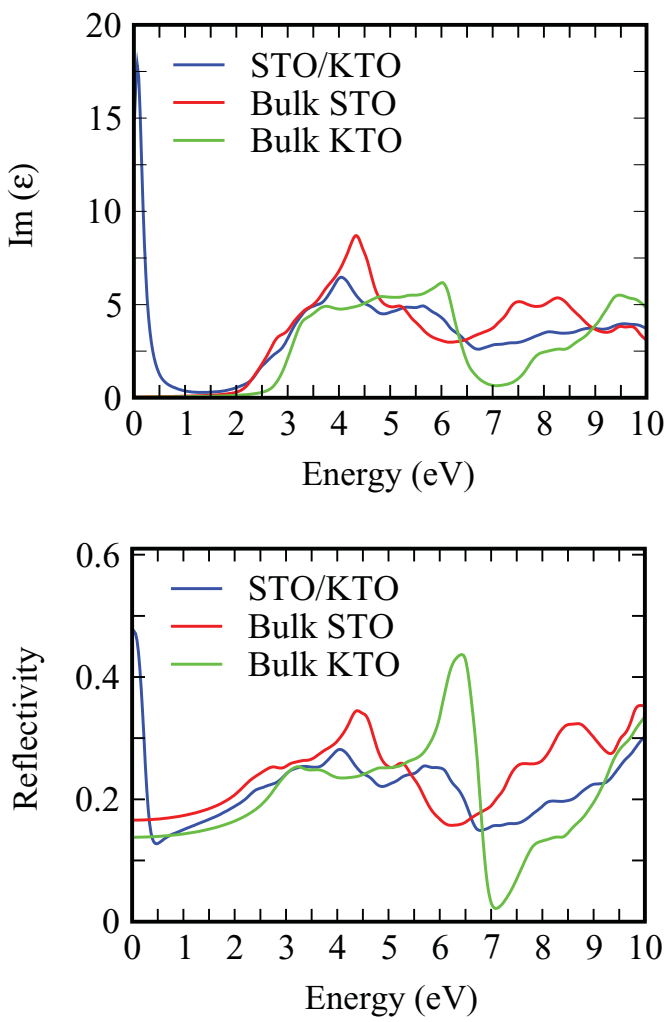

FIG. 3. (Color online) Imaginary part of the dielectric function as well as reflectivity of the $\mathrm{KTO} / \mathrm{STO}$ interface, as compared to bulk KTO and STO.

insulating behavior. The reflectivity spectrum obtained for the KTO/STO supercell is very similar to that of bulk STO, except for the low-energy region, where we observe the Drude peak for the interface system. A very similar behavior is seen in the $\operatorname{Im}(\epsilon)$ spectra. The two bulk materials exhibit band gaps, while the KTO/STO data reflect the metallic nature of the interface. The availability of the low-energy states for optical transitions reflects the energetic shifts of the Ti $3 d$, Ta $5 d$, and $\mathrm{O} 2 p$ states, as described before. The reflectivity spectrum of the KTO/STO supercell shows two broad peaks at energies of about 4 and 6 $\mathrm{eV}$. The remainders of these peaks are also seen in the $\operatorname{Im}(\epsilon)$ spectrum. The appearance of the peaks is expected because the band gaps differ in STO and KTO by $\sim 2 \mathrm{eV}$. Consequently, we can identify them with the optical transition maxima of STO and KTO at 4.5 and $6.5 \mathrm{eV}$, respectively. The slight shift in the peak positions is a relic of the finite size of the supercell used in the calculations.

In summary, we have studied the electronic and optical properties of the KTO/STO heterointerface by full-potential band structure calculations. Due to an almost ideal lattice match between the component materials, the structural optimization of the interface yields only minor alterations of the chemical bonding. Whereas KTO and STO are band insulators in the bulk phase, we find that the interface develops a metallic state, which agrees with experimental results for KTO thin films deposited on STO. ${ }^{22}$ Importantly, metallicity in the present case is obtained even without taking into account $\mathrm{O}$ vacancies or other kinds of structural defects. However, vacancies could still improve the metallicity. Analysis of the 
site-projected densities of states indicates that the metallic interface states trace back to the $\mathrm{O} 2 p$ and Ti $3 d$ orbitals. The creation of these states can be explained in terms of a weakening of the bonding between the transition metal and $\mathrm{O}$ atoms across the interface, which induces changes in the orbital occupations of the involved atoms.

The charge transfer is restricted to a very narrow layer of about $12 \AA$ thickness, which is also reflected by calculated optical reflectivity spectra. The spectra comprise the metallic Drude peak as well as the optical transitions of the component materials. In general, the relaxation of the electronic structure at the KTO/STO interface is found to be less complex than reported for the $\mathrm{LAO} / \mathrm{STO}$ interface, which is a consequence of the improved lattice match. Being released from the complications coming along with a strong structural relaxation, the KTO/STO interface gives rise to a prototypical system that can help to better understand the physics of related heterointerfaces, such as LAO/STO. A thorough experimental characterization of the KTO/STO system is thus expected to be very fruitful. *udo.schwingenschlog1@kaust.edu.sa

${ }^{1}$ M. Imada, A. Fujimori, and Y. Tokura, Rev. Mod. Phys. 70, 1039 (1998).

${ }^{2}$ H. Kroemer, Rev. Mod. Phys. 73, 783 (2001).

${ }^{3}$ A. Ohtomo and H. Y. Hwang, Nature (London) 427, 423 (2004).

${ }^{4}$ U. Schwingenschlögl and C. Schuster, Europhys. Lett. 86, 27005 (2009); Chem. Phys. Lett. 467, 354 (2009); Europhys. Lett. 81, 17007 (2008).

${ }^{5}$ G. Herranz, M. Basletić, M. Bibes, C. Carrétéro, E. Tafra, E. Jacquet, K. Bouzehouane, C. Deranlot, A. Hamzić, J.-M. Broto, A. Barthélémy, and A. Fert, Phys. Rev. Lett. 98, 216803 (2007).

${ }^{6}$ W. Siemons, G. Koster, H. Yamamoto, W. A. Harrison, G. Lucovsky, T. H. Geballe, D. H. A. Blank, and M. R. Beasley, Phys. Rev. Lett. 98, 196802 (2007).

${ }^{7}$ N. Reyren, S. Thiel, A. D. Caviglia, L. F. Kourkoutis, G. Hammerl, C. Richter, C. W. Schneider, T. Kopp, A.-S. Rütschi, D. Jaccard, M. Gabay, D. A. Muller, J.-M. Triscone, and J. Mannhart, Science 317, 1196 (2007).

${ }^{8}$ A. Dubroka, M. Rössle, K. W. Kim, V. K. Malik, L. Schultz, S. Thiel, C. W. Schneider, J. Mannhart, G. Herranz, O. Copie, M. Bibes, A. Barthélmy, and C. Bernhard, Phys. Rev. Lett. 104, 156807 (2010).

${ }^{9}$ M. Ben Shalom, M. Sachs, D. Rakhmilevitch, A. Palevski, and Y. Dagan, Phys. Rev. Lett. 104, 126802 (2010).

${ }^{10}$ B. Liu and X. Hu, Phys. Rev. B 81, 144504 (2010).

${ }^{11}$ T. Fix, F. Schoofs, J. L. MacManus-Driscoll, and M. G. Blamire, Phys. Rev. Lett. 103, 166802 (2009).

${ }^{12}$ C. Bell, S. Harashima, Y. Kozuka, M. Kim, B. G. Kim, Y. Hikita, and H. Y. Hwang, Phys. Rev. Lett. 103, 226802 (2009).

${ }^{13}$ C. Bell, S. Harashima, Y. Hikita, and H. Y. Hwang, Appl. Phys. Lett. 94, 222111 (2009).
${ }^{14}$ H. Chen, A. M. Kolpak, and S. Ismail-Beigi, Adv. Mater. 22, 2881 (2010).

${ }^{15}$ U. Schwingenschlögl and C. Schuster, Phys. Rev. Lett. 102, 227002 (2009); Appl. Phys. Lett. 90, 192502 (2007); Europhys. Lett. 77, 37007 (2007).

${ }^{16}$ U. Hiromoto and T. Sakudo, J. Phys. Soc. Jpn. 38, 183 (1975).

${ }^{17}$ M. E. Lines and A. Glass, Principles and Applications of Ferroelectric and Related Materials (Clarendon, Oxford, 1977).

${ }^{18}$ K. van Benthem, C. Elsäser, and R. H. French, J. Appl. Phys. 90, 6156 (2001).

${ }^{19}$ S. M. Shapiro, J. D. Axe, and G. Shirane, Phys. Rev. B 6, 4332 (1972).

${ }^{20}$ K. A. Müller and W. Berlinger, Phys. Rev. Lett. 26, 13 (1971).

${ }^{21}$ J. B. Goodenough, Rep. Prog. Phys. 67, 1915 (2004).

${ }^{22}$ A. Kalabukhov, R. Gunnarsson, T. Claeson, and D. Winkler, e-print arXiv:0704.1050.

${ }^{23}$ P. Hohenberg and W. Kohn, Phys. Rev. 136, B864 (1964).

${ }^{24}$ P. Blaha, K. Schwarz, G. Madsen, D. Kvasicka, and J. Luitz, WIEN2K, An Augmented Plane Wave + Local Orbitals Program for Calculating Crystal Properties (Technical University of Vienna, 2001).

${ }^{25}$ S. Cabuk, Phys. Status Solidi B 247, 93 (2010).

${ }^{26}$ M. Djermouni, A. Zaoui, S. Kacimi, and B. Bouhafs, Comput. Mater. Sci. 25, 478 (2002).

${ }^{27}$ Ferroelectrics and Related Substances, Landolt-Börnstein: Numerical Data and Functional Relationships in Science and Technology, Vol. 36: Oxides, edited by Y. Shiozaki, E. Nakamura, and T. Mitsui (Springer-Verlag, Berlin, 2002).

${ }^{28}$ C. Lasota, C. Z. Wang, R. Yu, and H. Krakauer, Ferroelectrics 194, 109 (1997). 